\title{
Estágios de mudanca de comportamento relacionados à ativídade física em acadêmicos de Educação Física
}

\author{
Stages of behavior change related to \\ physical activity in college students of \\ Physical Education
}

Paloma Cidade Cordeiro ${ }^{1}$

Gaia Salvador Claumann ${ }^{1}$

Érico Felden Pereira'

Adriana Coutinho de Azevedo Guimarães ${ }^{1}$

Andreia Pelegrini

\section{RESUMO}

A análise dos estágios de mudança de comportamento relacionados à atividade física (EMCAF) é uma etapa importante no desenvolvimento de propostas de intervenção, tendo em vista a necessidade de recomendações específicas para cada estágio. Assim, este estudo objetivou analisar os fatores associados aos EMCAF em acadêmicos dos cursos de Bacharelado e Licenciatura em Educação Física. Participaram do estudo 301 acadêmicos (173 rapazes) de uma universidade pública de Florianópolis, SC. Foram coletadas informações sociodemográficas (sexo, idade, estado civil, turno de estudo, nível econômico, trabalho, jornada de trabalho), antropométricas (massa corporal e estatura autorreferidas), percepção de saúde e EMCAF (pré-contemplação, contemplação, preparação, ação e manutenção). Os indivíduos classificados nos estágios de ação e manutenção foram agrupados e considerados ativos fisicamente, enquanto aqueles nos estágios pré-contemplação, contemplação e preparação foram considerados insuficientemente ativos. Observou-se que os acadêmicos encontraram-se, em sua maioria, nos estágios de manutenção $(50,8 \%)$ e preparação (24,6\%). Quando os EMCAF foram dicotomizados em ativos e insuficientemente ativos, $38,5 \%$ dos acadêmicos foram classificados como insuficientemente ativos, com maior proporção deste grupo entre os acadêmicos do turno noturno em relação aos do matutino $(46,0 \%$ e $30,0 \%$, respectivamente; $\mathrm{p}<0,05)$. Os acadêmicos que estudavam no período noturno $(\mathrm{RP}=1,23 ; \mathrm{IC} 95 \%=1,08-1,41)$ e aqueles com percepção negativa de saúde $(\mathrm{RP}=1,33$; $\mathrm{IC} 95 \%=1,14-1,54)$ apresentaram probabilidade maior de serem insuficientemente ativos. Conclui-se, portanto, que os acadêmicos que estudam no período noturno e aqueles com percepção negativa de saúde estão mais expostos a níveis insuficientes de atividade física.

\section{PALAVRAS-CHAVE}

Atividade física; Educação Física; Comportamentos saudáveis; Ensino superior.

\begin{abstract}
The analysis of the stages of behavior change related to physical activity (SBCPA) it's an important step in the development of proposals for intervention, in view of the necessity of specific recommendations for each stage. Thus, this study aimed to analyze the associated factors with the SBCPA in college students of Baccalaureate and Degree Physical Education courses. Participated in the study 301 college students (173 men) from a public university of Florianopolis, SC. Demographic information (gender, age, marital status, period of study, economic status, work, workday), anthropometric (self-reported body weight e height), health perception and SBCPA (pre-contemplation, contemplation, preparation, action were collected and maintenance). Individuals classified in the action and maintenance stages were grouped and considered physically active, while those in the pre-contemplation, contemplation and preparation stages were considered insufficiently active. It was observed that the students are mostly in the maintenance stage (50.8\%) and preparation (24.6\%). When SBCPA were dichotomized into active and insufficiently active, $38.5 \%$ of students were classified as insufficiently active, with a higher proportion among students of Bachelor in relation to the Bachelor $(46.0 \%$ and 30.0 $\%$, respectively $p<0.05)$. The students who study at night $(O R=1.23,95 \%$; CI 1.08 to 1.41) and those with negative bealth perception (PR=1.33, 95\%; CI 1.14 to 1.54) had a higher probability of being insufficiently active. It was conclude, therefore, that the college students who study at night and those with negative bealth perception are more exposed to insufficient levels of physical activity.
\end{abstract}

\section{KEYWORDS}

Physical activity; Physical Education; Healthy behaviors; Higher education. 


\section{INTRODUÇÃO}

Os efeitos da atividade física na redução do desenvolvimento de doenças cardiovasculares e promoção de saúde por meio de hábitos mais saudáveis têm sido amplamente estudados ${ }^{1-4}$. Resultados do levantamento VIGITEL ${ }^{5}$ revelaram que a população brasileira, no período de 2006 a 2012, apresentou níveis elevados de inatividade física, de $29,8 \%$ a $59,9 \%$, e, apesar da crescente melhora nos últimos anos, cerca de $14 \%$ a $65,8 \%$ dos adultos brasileiros ainda não atingem os níveis recomendados de prática de atividades físicas ${ }^{4-7}$. Cabe destacar que ao longo dos anos da realização desta pesquisa o instrumento para avaliação da prática de atividades físicas sofreu algumas alterações, entretanto o conteúdo principal do mesmo permaneceu semelhante.

Em se tratando da população de estudantes universitários, estudos que utilizaram os estágios de mudança de comportamento para avaliar o nível de atividade física encontraram prevalências de $68,4 \%{ }^{3}$ de indivíduos insuficientemente ativos em estudantes de diferentes áreas de conhecimento, e, especificamente, em acadêmicos de Educação Física (EF), foram verificadas prevalências de $43,1 \%^{4}$ e $45,2 \%{ }^{8}$. Os baixos níveis de atividade física são preocupantes, tendo em vista a relação destes com o desenvolvimento de fatores de risco cardiovasculares $^{9-10}$, os quais comprometem de maneira significativa a qualidade de vida das pessoas. Ademais, quando se trata de estudantes universitários da área da saúde espera-se que os hábitos sejam diferenciados das demais áreas de conhecimento, principalmente por estes lidarem com questões relacionadas à promoção da saúde ${ }^{4}$. Especificamente, em acadêmicos de Educação Física a literatura especializada revela prevalências elevadas de níveis insuficientes de atividade física, os quais não atingem a quantidade mínima recomendada de 150 minutos semanais de prática de atividades físicas moderada e vigorosa ${ }^{6,11,12}$.

A realidade acadêmica é uma etapa importante na vida destes estudantes, pois muitos dos comportamentos adquiridos durante toda a vida podem ser modificados em decorrência das novas experiências vividas no meio universitário. Com o ingresso no ensino superior, espera-se que os acadêmicos mantenham ou adotem hábitos mais saudáveis no seu cotidiano, especialmente àqueles da área da saúde, e dentre esses, os de Educação Física, já que possuem na sua grade curricular disciplinas que fomentam a necessidade de prática de atividade física e hábitos saudáveis para uma melhor qualidade de vida. No entanto, pesquisas como as de Rigoni et al. ${ }^{4}$ e Madureira et al. ${ }^{3}$, demonstram que $16,3 \%$ a 23,6\% dos universitários de Educação Física apresentam sobrepeso, e $21,2 \%$ a $68,4 \%$ são insuficientemente ativos.

Os estágios de mudança de comportamento relacionados à atividade física (EMCAF) podem ser considerados uma etapa importante no desenvolvimento de propostas de intervenção, tendo em vista a possibilidade de recomendações específicas para cada estágio ${ }^{13}$. Pesquisas que objetivaram analisar os EMCAF foram conduzidas em acadêmicos de Educação Física ${ }^{3,4,11,14}$. Destaca-se, dentre os principais resultados, que apesar de a maioria dos acadêmicos serem classificados no estágio de manutenção e ação ${ }^{4,11}$, ainda, uma parcela elevada de acadêmicos são classificados como insuficientemente ativos, ou seja, encontram-se nos estágios pré-contemplação, contemplação e preparação ${ }^{3,11}$. Evidências apontam associação dos EMCAF com estado nutricional ${ }^{3}$, barreiras para a prática de atividade física ${ }^{4}$, sexo $^{7}$ e escolaridade materna ${ }^{8}$. Não obstante, é necessário verificar 
as possíveis associações entre os EMCAF com variáveis sociodemográficas (sexo, idade, turno de estudo, estado civil, trabalho, jornada de trabalho e nível econômico), status de peso (índice de massa corporal) e percepção de saúde, pois a partir destes achados, as estratégias de intervenção podem ser direcionadas para os grupos mais expostos visando às mudanças de comportamento. Neste sentido, o presente estudo teve como objetivo analisar os fatores associados aos EMCAF em acadêmicos dos cursos de Bacharelado e Licenciatura em Educação Física.

\section{METODOS}

Trata-se de uma pesquisa quantitativa, descritiva com delineamento transversal, a qual faz parte do projeto "Estudo longitudinal da saúde e cognição de acadêmicos de Educação Física da UDESC", aprovado pelo Comitê de Ética em Pesquisa com Seres Humanos.

Foram considerados elegíveis os acadêmicos matriculados nos cursos de Bacharelado e Licenciatura em Educação Física, no primeiro semestre de 2013, em uma universidade pública da cidade de Florianópolis - SC. No referido período 500 acadêmicos (253 de Bacharelado) estavam matriculados na instituição. A amostra mínima estimada utilizando um erro de quatro pontos percentuais, com prevalência de $50 \%$, foi de $274^{15}$. Foram acrescentados $10 \%$ para perdas amostrais, totalizando 301 acadêmicos. Aqueles que estavam presentes em sala de aula no momento da coleta de dados, que aceitaram participar voluntariamente e assinaram o Termo de Consentimento Livre e Esclarecido foram incluídos no estudo.

Foram coletados dados sociodemográficos por meio de uma ficha contendo informações referentes ao sexo (masculino, feminino), à idade (anos), a qual foi categorizada em $<20$ anos e $\geq 20$ anos, turno (diurno, em que estudam os acadêmicos do curso de Bacharelado, e noturno, em que estudam os acadêmicos da Licenciatura), estado civil (com companheiro, sem companheiro), trabalho (sim, não) e jornada de trabalho ( $<20$ horas semanais e $\geq 20$ horas semanais). O nível econômico foi investigado pelo questionário da Associação Brasileira de Empresas de Pesquisa (2010). As classes econômicas foram agrupas em alta (A1+A2+B1) e média/baixa (B2, C1, C2, D e E). Também foram coletadas a massa corporal e estatura autorreferidas ${ }^{16}$, para o cálculo do índice de massa corporal (IMC), a partir do qual os indivíduos foram classificados, quanto ao status do peso, em eutróficos e com excesso de peso (indivíduos com sobrepeso e obesidade) ${ }^{17}$.

A percepção de saúde foi verificada por meio da seguinte questão: "De um modo geral, como você considera o seu estado de saúde atual?”. A resposta foi apresentada em escala Likert, em que (1) corresponde a "muito ruim" e (5) a "muito bom". No presente estudo, as categorias "muito ruim", "ruim" e "regular" foram agrupadas e consideradas percepção negativa de saúde, enquanto as categorias "bom" e "muito bom", como percepção de saúde positiva ${ }^{18}$.

Os EMCAF foram verificados por intermédio de questionário, validado para uso em amostra de adultos jovens ${ }^{19-20}$, que obedecem a uma sequência: pré-contemplação (o sujeito não pretende modificar seu comportamento num futuro próximo), contemplação (existe a intenção de mudar, mas não imediatamente), preparação (existe a intenção de mudar nos próximos seis meses), ação (mudanças recentes no comportamento) e manutenção (as mudanças no comportamento já são mantidas por mais de seis meses). No presente estudo o comportamento 
avaliado foi a prática de atividades físicas. Para fins de análise, os EMCAF foram analisados nas cinco categorias e também foram agrupados em duas categorias: ativos (ação e manutenção) e insuficientemente ativos (pré-contemplação, contemplação e preparação), conforme categorização utilizada previamente em estudos semelhantes ${ }^{3,4,8}$. Solicitou-se aos acadêmicos que não considerassem as atividades realizadas durante as aulas do curso de Educação Física, já que para muitos deles são essas as atividades físicas moderadas que realizam.

Os dados foram analisados inicialmente pela estatística descritiva (média, desvio-padrão e distribuição de frequências). A normalidade dos dados foi verificada por meio do teste Kolmogorov-Smirnov. As possíveis associações entre as variáveis foram analisadas por meio do teste Qui-quadrado e/ou Exato de Fischer. Foi utilizada a regressão de Poisson obtendo razões de prevalência em modelos bruto e ajustado, que foram estimados por intervalos com $95 \%$ de confiança. As variáveis que apresentaram na análise bruta $\mathrm{p}<0,20$ permaneceram no modelo ajustado ${ }^{21}$. Considerou-se como desfecho "negativo" os indivíduos insuficientemente ativos e como desfecho "positivo" os ativos fisicamente. Em todas as análises adotou-se o nível de significância de 5\%, utilizando-se o programa estatístico SPSS, versão 20.0.

\section{RESULTADOS}

Participaram do estudo 301 acadêmicos dos cursos de Bacharelado e Licenciatura em Educação Física que responderam aos questionários adequadamente o equivalente a $60,2 \%$ da população alvo. Todos os acadêmicos elegíveis (que estavam presentes em sala de aula no momento da coleta) aceitaram participar do estudo, não havendo recusas e/ou preenchimento inadequado dos questionários. A média de idade da amostra foi de 23,1 anos. A maioria dos acadêmicos era do sexo masculino $(57,5 \%)$, estudava no período noturno $(53,5 \%)$, não tinha companheiro $(64,1 \%)$, pertencia à classe econômica média $(53,8 \%)$ e trabalhava $(76,1 \%)$ com jornada de trabalho maior ou igual a 20 horas por semana $(55,0 \%)$. Notou-se que, aproximadamente, um a cada quatro acadêmicos apresentava excesso de peso (24,1\%). Quanto aos EMCAF, verificou-se que $50,8 \%$ dos acadêmicos encontravam-se no estágio de manutenção (Tabela 1).

Quando os EMCAF foram agrupados em ativos e insuficientemente ativos, 38,6\% dos acadêmicos foram classificados como insuficientemente ativos, com maior proporção entre os acadêmicos do turno noturno em relação aos do turno matutino (46,0\% e 30,0\%, respectivamente; $\mathrm{p}<0,05)$. Ademais, observou-se que os acadêmicos encontravam-se, predominantemente, nos estágios de manutenção (50,8\%) e preparação (24,6\%) (Figura 1a).

$\mathrm{Na}$ comparação dos EMCAF entre os cursos (Figura 1b), diferenças significativas foram observadas nos estágios manutenção e contemplação, revelando maior proporção de acadêmicos do curso de Bacharelado $(57,1 \%)$ no estágio de manutenção em relação aos do curso de Licenciatura (45,3\%). Por outro lado, maior proporção de acadêmicos do curso de Licenciatura se encontra no estágio de contemplação (17,4\%) em comparação aos do curso de Bacharelado (7,9\%).

A Tabela 2 apresenta a associação dos EMCAF com as variáveis independentes. Observou-se, na análise bruta, associação do curso e percepção de saúde com os EMCAF. No modelo ajustado, as variáveis curso e percepção de saúde permaneceram associadas ao desfecho, revelando que os acadêmicos da 
Licenciatura apresentam uma probabilidade maior de 1,24 (IC95\%=1,09$1,41)$ de serem insuficientemente ativos e aqueles com percepção negativa de saúde apresentaram uma probabilidade maior de 1,33 (IC95\%=1,14-1,54) de serem insuficientemente ativos quando comparados aos seus pares do Bacharelado e com percepção de saúde positiva.

TABELA 1 - Média (desvio padrão) e distribuição de frequência das variáveis investigadas nos acadêmicos dos cursos de Educação Física, de acordo com o sexo. Florianópolis, SC. 2013.

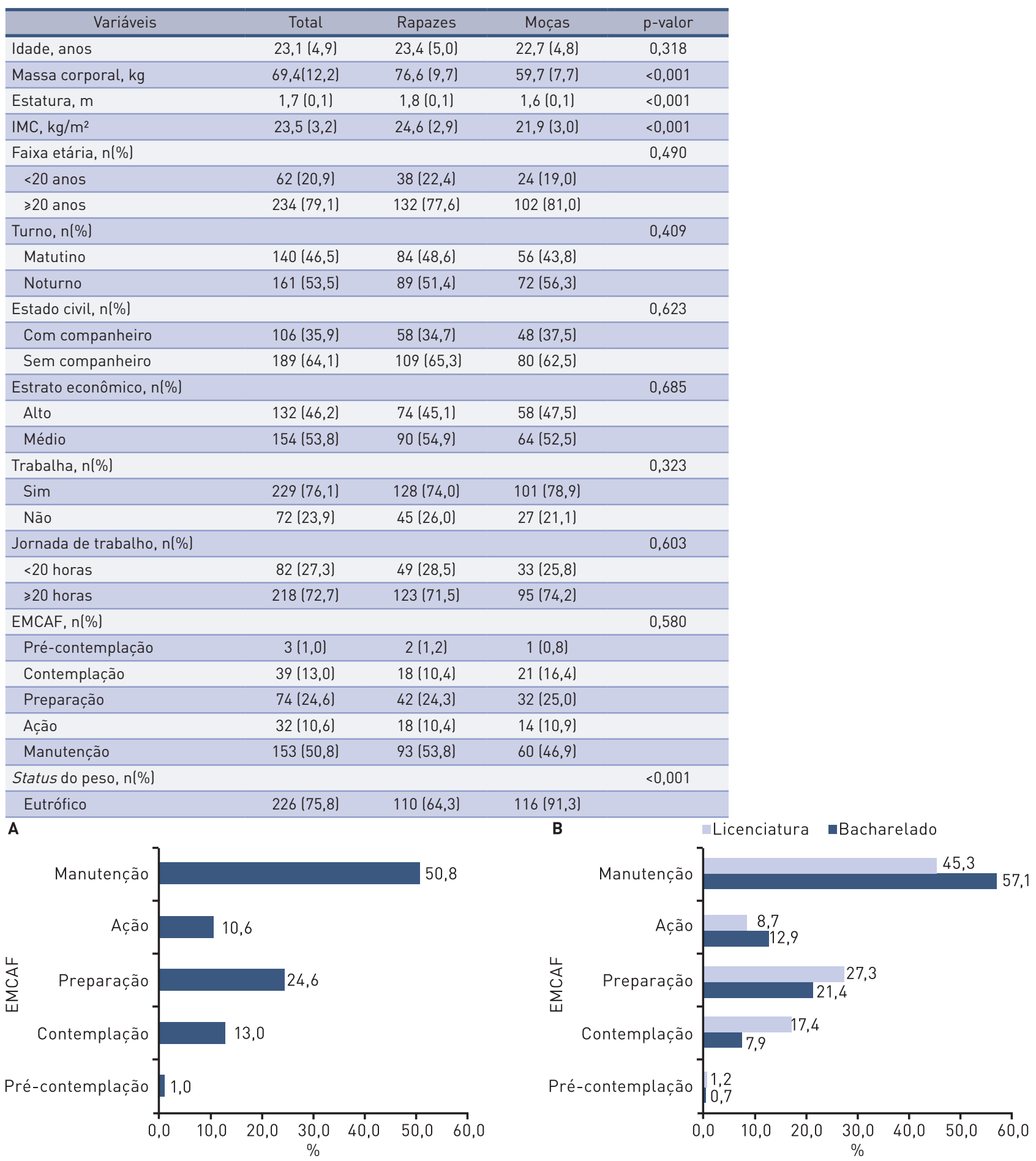

FIGURA 1 - Distribuição dos acadêmicos em relação ao estágio de mudança de comportamento relacionados à atividade física geral (a) e por habilitação (b). * $p<0,05$ (teste para diferenças entre duas proporções) 
IMC: índice de massa corporal; EMCAF: estágios de mudança de comportamento relacionados à atividade física; kg: quilogramas; m: metros; \%: frequência relativa.

TABELA 2 - Análise de regressão bruta e ajustada entre EMCAF e as variáveis independentes. Florianópolis, SC. 2013.

\begin{tabular}{|c|c|c|c|c|}
\hline Variáveis & RP (IC95\%) & p-valor & RP (IC95\%)** & p-valor \\
\hline \multicolumn{5}{|l|}{ Sexo } \\
\hline Masculino & $0,94(0,84-1,05)$ & 0,264 & Excluído & - \\
\hline Feminino & 1 & & & \\
\hline \multicolumn{5}{|l|}{ Faixa etária } \\
\hline$<20$ anos & 1 & & & \\
\hline$\geqslant 20$ anos & $1,06(0,93-1,21)$ & 0,388 & Excluído & - \\
\hline \multicolumn{5}{|l|}{ Turno } \\
\hline Matutino & 1 & & 1 & \\
\hline Noturno & $1,17(1,05-1,31)$ & 0,004 & $1,24(1,09-1,41)$ & 0,002 \\
\hline \multicolumn{5}{|l|}{ Estado civil } \\
\hline Sem companheiro & $0,98(0,87-1,10)$ & 0,728 & Excluído & - \\
\hline Com companheiro & 1 & & & \\
\hline \multicolumn{5}{|l|}{ Estrato econômico } \\
\hline Alto & 1 & & 1 & 0,400 \\
\hline Médio & $1,09(0,97-1,21)$ & 0,157 & $1,06(0,93-1,21)$ & \\
\hline \multicolumn{5}{|l|}{ Trabalha } \\
\hline Sim & $0,96(0,84-1,09)$ & 0,535 & Excluído & - \\
\hline Não & 1 & & & \\
\hline \multicolumn{5}{|l|}{ Jornada de trabalho } \\
\hline$<20$ horas & 1 & & Excluído & - \\
\hline$\geqslant 20$ horas & $0,98(0,86-1,11)$ & 0,732 & & \\
\hline \multicolumn{5}{|l|}{ Percepção de saúde } \\
\hline Positiva & 1 & & 1 & \\
\hline Negativa & $1,32(1,14-1,52)$ & $<0,001$ & $1,33(1,14-1,54)$ & $<0,001$ \\
\hline \multicolumn{5}{|l|}{ Status do peso } \\
\hline Eutrófico & 1 & 0,342 & Excluído & - \\
\hline Excesso de peso & $1,07(0,94-1,21)$ & & & \\
\hline
\end{tabular}

** análise ajustada pelas variáveis que apresentaram $\mathrm{p}<0,20$ na análise bruta.

\section{DISCUSSÃO}

O presente estudo identificou que a maior parte dos acadêmicos dos cursos de Educação Física encontrava-se no estágio de manutenção. Este resultado é semelhante ao estudo de Guedes ${ }^{11}$, o qual apontou que $54 \%$ dos universitários de Londrina/PR se encontravam no mesmo estágio. Estudo conduzido em acadêmicos de diferentes cursos de uma universidade pública de Florianópolis/SC revelou que $61,4 \%$ destes se encontravam no estágio de manutenção ${ }^{3}$. Estes resultados são importantes, pois indicam que a maior parte dos acadêmicos já apresenta um comportamento regularmente ativo há mais de seis meses. Neste sentido, manter um ambiente atrativo e agradável estimula a priorização da prática e consequentemente a adoção e manutenção de um estilo de vida ativo fisicamente ${ }^{3}$.

Outro estágio no qual esteve classificada boa parte dos estudantes é o de preparação, ou seja, estes ainda não estão envolvidos em alguma atividade fí- 
sica, mas desejam, em um futuro próximo, começar a praticá-la regularmente. Resultados similares foram encontrados por Madureira et al. ${ }^{3}$ ao analisarem os EMCAF em acadêmicos de vários cursos de uma universidade de Santa Catarina (29,5\%). Assim, programas de intervenção de AF são fundamentais para consolidar a escolha pela prática regular ${ }^{13}$. Desta maneira, programas permanentes de AF para a comunidade são necessários, para que os indivíduos que se encontram neste estágio sintam-se incentivados a exercer um estilo de vida ativo fisicamente. Destaca-se a necessidade de os estudantes que ainda não se encontram nos estágios fisicamente ativos adotarem uma atitude diferenciada em relação à atividade física, encarando-a também como um compromisso importante de sua rotina ${ }^{4}$. Oliveira ${ }^{22}$ sugere a necessidade de intervenção por meio do desenvolvimento de programas de promoção de atividade física, fornecendo sustentação para que as intervenções sejam planejadas de acordo com as particularidades regionais da população alvo. Isto, sabendo que as características locais que influenciam a prática de atividades físicas podem ser diferentes daquelas identificadas para a população como um todo.

Apesar de a maior parte dos acadêmicos do presente estudo se apresentar nos estágios de manutenção e ação, muitos $(38,6 \%)$ ainda se encontram nos estágios de pré-contemplação, contemplação e preparação. Estes resultados corroboram os dados encontrados por Rigoni et al. ${ }^{4}$ e Silva et al. ${ }^{6}$, os quais constataram que 43,1\% e 45,2\% dos acadêmicos de Educação Física, respectivamente, estavam nos estágios menos ativos fisicamente (pré-contemplação, contemplação e preparação). Neste contexto, apesar de serem acadêmicos de Educação Física e terem conhecimento sobre os benefícios de um comportamento de vida fisicamente ativo, estes ainda não conseguem atingir as recomendações de, no mínimo, 150 minutos semanais de atividade física de intensidade moderada e vigorosa, de forma contínua ou acumulada ${ }^{23}$.

$\mathrm{Na}$ literatura pesquisada, encontrou-se poucos estudos que verificaram associação do EMCAF com o curso em Educação Física ${ }^{4,6,11}$, os quais afirmam que os níveis de atividade física dos acadêmicos são superiores aos da população em geral e complementam que essa condição pode ser explicada pelo histórico esportivo e perfil dos alunos que ingressam nesse curso.

Para interpretar os resultados encontrados no presente estudo, devem ser levados em consideração, também, os períodos em que os cursos são ministrados, sendo o curso de Bacharelado ministrado pela manhã e o de Licenciatura, à noite. Verificou-se que os acadêmicos de Bacharelado em Educação Física apresentavam comportamento mais ativo fisicamente em relação aos do curso de Licenciatura, os quais, muitas vezes, escolhem o curso por ser no período noturno e possibilitar a inserção no mercado de trabalho durante o dia. Neste sentido, os níveis insuficientes de atividade física observados nos acadêmicos do curso de Licenciatura podem ser explicados pelo fato de que esses acadêmicos, em sua maioria, já estão inseridos no mercado de trabalho com jornada superior a 20 horas semanais (62,5\%). Por outro lado, apenas 29,3\% dos acadêmicos do curso de Bacharelado em Educação Física trabalham e destes, 46,4\% apresentam jornada de trabalho superior a 20 horas semanais (dados não apresentados).

Níveis insuficientes de atividade física durante o período universitário podem, e devem, ser uma das questões priorizadas na universidade. É bastante provável que acadêmicos de Educação Física tenham interesse em adotar 
e manter hábitos de vida saudáveis, de modo geral, mas especialmente em relação à prática de atividades físicas e, aqueles que não estão conseguindo alcançar estes objetivos podem estar enfrentando algumas barreiras e merecem atenção ${ }^{24}$. Assim, o próprio ambiente universitário deve não apenas incentivar tais hábitos, como possibilitar iniciativas de promoção da saúde proporcionando melhorias na qualidade de vida dos universitários no presente momento, visando, também, a continuidade disso após o término da graduação.

Os resultados do presente estudo indicaram associação dos EMCAF com a percepção de saúde dos acadêmicos, apontando que os indivíduos com percepção negativa de saúde apresentaram maior probabilidade de serem classificados como insuficientemente ativos. Esses resultados divergem dos encontrados em acadêmicos de Educação Física do estado de Sergipe, em que os autores não verificaram associação entre atividade física e percepção de saúde ${ }^{8}$. Por outro lado, em uma revisão sistemática envolvendo adolescentes, foi verificada uma associação direta entre a prática de atividade física e percepção de saúde ${ }^{25}$. Esta associação pode ser fundamentada por fatores fisiológicos e psicossociais, em virtude da repercussão que a atividade física possui, por exemplo, na atenuação da dor, favorecendo a sensação de bem-estar para o indivíduo, além dos persuasivos efeitos na saúde mental, melhoria da imagem corporal e redução dos sintomas de ansiedade e depressão, podendo assim gerar uma percepção mais positiva de saúde $26-27$.

O presente estudo apresenta limitações, como: a) o delineamento transversal, que impede maiores conclusões de causa e efeito entre as variáveis, principalmente relacionado a atividade física e percepção de saúde; b) a amostra do estudo consistiu em um grupo de indivíduos particularmente envolvidos em práticas habituais de atividade física e esportivas (acadêmicos dos cursos de Educação Física), o que, por sua vez, limita a extrapolação dos resultados para universitários de outras áreas do conhecimento; c) utilização de medidas autorreferidas, que impedem uma afirmação em sua totalidade, já que dependem da fidedignidade dos participantes.

Em conclusão, mais da metade dos participantes do presente estudo se encontrava nos estágios de manutenção e ação, revelando que os acadêmicos desta universidade são ativos fisicamente. Apesar disso, grande parcela ainda apresenta comportamento insuficientemente ativo (pré-contemplação, contemplação e preparação). Os grupos com maiores probabilidades de níveis insuficientes de atividade física foram os acadêmicos do turno noturno e àqueles com percepção negativa de saúde.

Desta forma, recomenda-se que a instituição investigada elabore estratégias no sentido de estimular que todos os acadêmicos de Educação Física, principalmente aqueles insuficientemente ativos, atinjam às recomendações para atividade física, e os ativos, mantenham o seu envolvimento nas atividades físicas. A universidade pode auxiliar esses acadêmicos oferecendo atividades extracurriculares que incentivem a adoção do comportamento ativo, disponibilizando locais seguros de prática e estimulando a participação coletiva. Conhecer o perfil da atividade física nos diferentes contextos do cotidiano dos indivíduos é um passo importante para o desenvolvimento de políticas públicas de saúde. Sugere-se que estudos com delineamento longitudinal sejam conduzidos com o objetivo de verificar as escolhas e motivações para os EMCAF. 


\section{REFERÊNCIAS}

1. Hallal PC et al. Evolução da pesquisa epidemiológica em atividade física no Brasil: revisão sistemática. Rev Saúde Pública. 2007; 41(3): 453-60.

2. Haskell WL et al. Physical activity and public health: updated recommendation for adults from the American College of Sports Medicine and the American Heart Association. Circulation. 2007; 116(9): 1081-93.

3. Madureira AS et al. Associação entre estágios de mudança de comportamento relacionados à atividade física e estado nutricional em universitários. Cad Saúde Pública. 2009; 25(10): 2139-46.

4. Rigoni PAG et al. Estágios de mudança de comportamento e percepção de barreiras para a prática de atividade física em universitários do curso de Educação Física. Rev Bras Ativ Fís Saúde. 2012; 17(2): 87-92.

5. Vigitel. Vigilância de Fatores de risco e Proteção para doenças crônicas por inquérito telefônico. Ministério da Saúde: Secretaria de Vigilância em Saúde /MS. 2012.

6. Brasil. IBGE, Instituto Brasileira de Geografia e estatística. 2011; Brasil. Disponível em: http://www.ibge.gov.br/home/

7. Silva DAS et al. Estágios de mudanças de comportamento para atividade física em estudantes de uma cidade do Brasil. Rev Salud Pública. 2010; 12(4): 623-34.

8. Silva DAS, Pereira IMM. Estágios de mudança de comportamento para atividade física e fatores associados em acadêmicos de educação física. Rev Bras Ativ Fís Saúde. 2010; 15(1): $15-20$.

9. Jeon CY, Lokken RP, Hu FB, Van Dam RM. Physical activity of moderate intensity andrisk of type 2 diabetes: a systematic review. Diabetes Care. 2007; 30(3): 744-52.

10. Williams PT, Thompson PD. Walking Versus Running for Hypertension, Cholesterol, and Diabetes Mellitus Risk Reduction. Arterioscler Thromb Vasc Biol. 2013; 33: 1085-91.

11. Guedes DP, Santos CA, Lopes CC. Estágios de mudança de comportamento e prática habitual de atividade física em universitários. Rev Bras Cineantropom Desempenho Hum. 2006; 8(4): 5-15.

12. Prates MEF. Impacto da disciplina de qualidade de vida na mudança de comportamento para prática de atividades físicas em estudantes universitários [dissertação de mestrado]. Florianópolis: Universidade Federal de Santa Catarina; 2004.

13. Markus BH, Simkin LR. The transtheoretical model: applications to exercise behavior. Med Sci Sports Exerc. 1994; 26: 1400-4.

14. Silva DAS. Nível de atividade física e fatores associados em acadêmicos de educação física de uma universidade pública do nordeste do Brasil. Rev Bras Ativ Fís Saúde. 2011; 17(1): 87-92.

15. Rodrigues, PC. Bioestatística. Niterói: Editora da Universidade Federal Fluminense, 2002.

16. Coqueiro RS et al. Medidas auto-referidas são válidas para avaliação do estado nutricional na população brasileira? Rev Bras Cineantropom Desempenho Hum. 2009; 11(1): 113-9.

17. World Health Organization, WHO. Obesity: Preventing and Managing the Global Epidemic. Report of a WHO Consultation on Obesity. Geneva, 2002.

18. Martins MO, Petroski, EL. Mensuração da percepção de barreiras para a prática de atividades físicas: uma proposta de instrumento. Rev Bras Cineantropom Desempenho Hum. 2000; 2(1): 58-65.

19. Plotnkoff RC, Hotz SB, Birkett NJ, Courneya KS. Exercise and the transtheoretical model: a longitudinal test of a population sample. Prev Med. 2001; 33: 441-52.

20. WHO; World Health Organization. Global recommendations on physical activity for health, 2011.

21. Maldonado G, Greenland S. Simulation study of confounder-selection strategies. Am J Epidemiol. 1993; 138(11): 923-36.

22. Oliveira NKR et al. Estágios de mudança de comportamento para a atividade física em adolescentes. Motriz: Rev Educ Fís. 2012; 18(1): 42-54.

23. Nahas MV. Atividade física, saúde e qualidade de vida. Londrina: Midiograf, 2001.

24. Sousa TF, Santos SFS, José HPM. Barreiras percebidas à prática de atividade física no nordeste do Brasil. Rev Pensar a Prática. 2010; 13(1): 1-15. 
25. Luiz A et al. Associação entres atividade física e percepção de saúde em adolescentes: revisão sistemática. Rev Bras Ativ Fís Saúde. 2011; 16(3): 246-54.

26. Nesheim $T$ et al. Physical activity and perceived health among 11-15-year old Norwegians. Tidsskr Nor Laegeforen. 2003; 6(123): 772-4.

27. Paluska SA, Schwenk TL. Physical activity and mental health: current concepts. Sports Med. 2000; 29: 167-80.

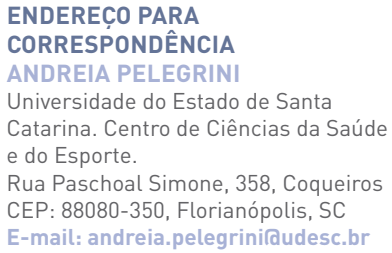

